\title{
Hábitat de alimentación del carpintero negro (Campephilus magellanicus) en ecosistemas forestales del parque nacional Nahuelbuta, región de La Araucanía, Chile
}

\author{
Feeding habitat of the Magellanic woodpecker (Campephilus magellanicus) across forest \\ ecosystems at Nahuelbuta National Park, La Araucanía Region, Chile
}

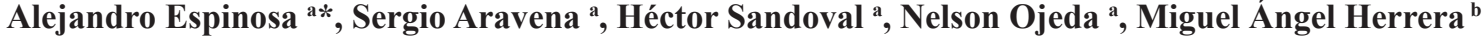 \\ *Autor de correspondencia: ${ }^{a}$ Universidad de La Frontera, Departamento de Ciencias Forestales, Temuco, Chile, \\ tel.: 56-45-2325661, alejandro.espinosa@ufrontera.cl \\ ${ }^{\mathrm{b}}$ Universidad de Córdoba, Departamento de Ingeniería Forestal, España.
}

\begin{abstract}
SUMMARY
In this study we described the feeding habitat of the Magellanic woodpecker (Campephilus magellanicus) across forest ecosystems at Nahuelbuta National Park ( $37^{\circ} 48^{\prime} \mathrm{S}$ y $\left.72^{\circ} 59^{\prime} \mathrm{W}\right)$, La Araucanía region, Chile. The study aims at filling the gaps on base line and scientific information needed for the elaboration of native forest management schemes oriented to the protection of wild fauna. Environmental and vegetation attributes related to the presence of $C$. magellanicus were identified in two samples applied: October 2008 and March 2009. Fifty count spots with three replications in each were sampled. We estimated population attributes of the bird assemblage from which $C$. magellanicus is an integral part. We identified 30 bird species and 4 forest types associated with the habitat of C. magellanicus. The forest types are combinations of Chilean birch species as "coigüe" (Nothofagus dombeyi), "lenga" (N. pumilio) and ñirre (N. antarctica), and monkey puzzle tree "araucaria" (Araucaria araucana). The forest types are "coigüearaucaria", "coigüe-araucaria-lenga", and "coigüe" and "ñirre". We found significant correlations between C. magellanicus and crown-cover of dominant trees in 2008 and 2009, herbaceous-cover in 2009 and a very significant correlation with the decay degree of standing trees and herbaceous-cover in 2008. We concluded that the three most correlated attributes with the presence of C. magellanicus on the study area were the crown-cover of dominant trees, herbaceous-cover and the decay degree of standing trees.
\end{abstract}

Key words: forest structure, forest composition, feeding habitat, bird assemblage, bird population parameters.

\section{RESUMEN}

El presente estudio describe el hábitat de alimentación del carpintero negro (Campephilus magellanicus) en ecosistemas boscosos del parque nacional Nahuelbuta ( $37^{\circ} 48^{\prime} \mathrm{S}$ y $72^{\circ} 59^{\prime} \mathrm{O}$ ), región de La Araucanía, Chile. Estuvo orientado a llenar parte de los vacíos que existen en información científica y de línea base necesaria para elaborar planes de manejo de bosque nativo orientados a la protección de la fauna silvestre. En dos muestras, compuestas por 50 estaciones de escucha cada una, con tres repeticiones, desarrolladas en octubre de 2008 y marzo de 2009, se evaluaron atributos medioambientales y vegetacionales relacionados con la presencia de C. magellanicus. También se estimaron atributos poblacionales del ensamblaje avifaunístico del cual C. magellanicus es parte integral. Se identificaron 30 especies de aves y 4 tipos de bosques asociados a la presencia de C. magellanicus. Los tipos de bosque corresponden a asociaciones de especies del género Nothofagus, como coigüe ( $N$. dombeyi), lenga ( $N$. pumilio) y ñirre ( $N$. antarctica), así como también araucaria (Araucaria araucana), las cuales constituyen bosques de "coigüe-araucaria", "coigüe-araucaria-lenga", "coigüe" y "ñirre". Se encontraron correlaciones significativas entre la presencia de C. magellanicus y la cobertura de los árboles dominantes en 2008 y 2009 y una correlación muy significativa con el estado de descomposición de los árboles en pie y la cobertura herbácea en 2008. Se concluyó que las tres variables más correlacionadas con la presencia de C. magellanicus fueron la cobertura de los árboles dominantes, la cobertura herbácea y el estado de descomposición de los árboles en pie.

Palabras clave: estructura de bosques, composición de bosques, hábitat de alimentación, ensamble avifaunístico, parámetros poblacionales avifaunísticos.

\section{INTRODUCCIÓN}

Campephilus magellanicus (King 1828) es una especie endémica de Chile y Argentina (Rozzi et al. 1995). Debido a la susceptibilidad que poseen los carpinteros a cambios en el hábitat en que viven, se les considera como especies indicadoras de biodiversidad de los ecosistemas forestales $\mathrm{y}$, más específicamente, como indicadores de biodiversidad de avifauna en los bosques (Mikusinski et al. 2001, Virkkala 2006). Su estado de conservación corresponde a preocupación menor (IUCN 2014). La importancia ecológica de esta especie es múltiple y es considerada una espe- 
cie "paraguas", debido al extenso ámbito de hogar que posee. Como consecuencia de ello, al brindársele protección, se conservan comunidades bióticas completas (Arango et al. 2007). Este mismo hecho hace que también sea considerada como una especie "carismática" o "emblemática", atrayendo la atención y admiración del público, por ende, se puede usar como especie central en actividades de educación ambiental o ecoturismo (Walpole y Leader-William 2002). Se le considera además una especie "clave", porque al hacer excavaciones en los árboles, ya sea para alimentarse, dormir o anidar, crea hábitats favorables para otras especies que usan cavidades secundarias (Ojeda 2004, Drever et al. 2008).

Existen diversos artículos científicos que hacen énfasis en aspectos biológicos, ecológicos y etológicos de C. magellanicus (Short 1970, Ojeda 2003, 2004, Vergara y Schlatter 2004, 2006, Ojeda y Chazarreta 2006, Ojeda et al. 2007, Arango et al. 2007, Chazarreta et al. 2011), en cambio, Arango et al. (2007) utilizan una aproximación biocultural con el fin de fomentar su conservación en Tierra del Fuego. Se necesita urgentemente investigar acerca de esta especie, pues los bosques nativos que componen su hábitat están siendo sometidos a una fuerte fragmentación y empobrecimiento ecológico. Como consecuencia de ello, se generan devastadores procesos de aislamiento y pérdida de conectividad entre las poblaciones de flora y fauna que habitan tales fragmentos, amenazando la viabilidad y sobrevivencia de especies como C. magellanicus, máxime si se considera que ya presenta algún grado de amenaza (Willson et al. 1994, Aguayo et al. 2009).

Pese al extraordinario rol que juega C. magellanicus, hasta la fecha muy pocos artículos publicados en Chile que integren conocimientos generados sobre una base comprensiva y con perspectivas de aplicación. Se distingue, por ejemplo, una falta de integración entre la información ecológica, etológica y biológica de la especie con aquella generada desde las ciencias forestales, existiendo una brecha del conocimiento que es importante remediar. Por ejemplo, se desconocen las variables del hábitat asociadas directamente con la ocurrencia de individuos de C. magellanicus y cómo esta información puede ser utilizada para diseñar pautas de manejo forestal orientado a la conservación de esta especie. Existe evidencia a nivel mundial que, al no asumir a tiempo y de buena forma este tipo de tareas, incluso aplicando sistemas silviculturales protectores más modernos, se corre el peligro de perder para siempre elementos clave de los bosques nativos - con todas sus consecuencias ecológicas. Tal es el caso, por ejemplo, de la especie europea conocida como carpintero de dorso blanco (Dendrocopus leucoto Bélkő, 1976), la que se encuentra en peligro de extinción, ampliamente documentado en el ámbito científico (Angelstam y Mikusinski 1994, Czeszczewik y Walankiewicz 2006).

Contar con una buena base de conocimiento acerca del uso del hábitat de alimentación, es un primer paso esencial para diseñar medidas de manejo forestal adecuadas para conservar especies de avifauna que dependen de la existencia de ecosistemas forestales nativos en buen estado de conservación. En este contexto, el presente estudio plantea como hipótesis que al interior de los ecosistemas forestales del parque nacional Nahuelbuta, existen atributos estructurales de la vegetación que se relacionan directamente con el hábitat de alimentación de C. magellanicus, los cuales, además, no presentan variaciones estacionales. Consecuentemente, el objetivo del presente estudio consiste en identificar aquellos atributos estructurales de la vegetación correlacionados con el hábitat de alimentación de C. magellanicus y establecer la existencia de variaciones estacionales entre estos.

\section{MÉTODOS}

Área de estudio. El estudio se desarrolló en el parque nacional Nahuelbuta, ubicado en las regiones del Biobío y de La Araucanía, entre 800 y 1.550 m s.n.m. (punto más alto de la Cordillera de Nahuelbuta). Este parque se encuentra en las provincia de Arauco (región del Biobío) y Malleco (región de La Araucanía); más específicamente, en las comunas de Cañete (región del Biobío), Angol y Purén (región de La Araucanía), entre los $37^{\circ} 44^{\prime}$ a $37^{\circ} 51^{\text {' }}$ de latitud sur y $72^{\circ} 55^{\prime}$ a $73^{\circ} 03^{\prime}$ de longitud oeste.

Método de muestreo. Mediante avistamiento y/o escucha de las aves, se diseñó un muestreo representativo de dos condiciones estacionales: primavera (octubre de 2008) y verano (marzo 2009). En ambos sitios de muestreo, se evaluó la presencia de C. magellanicus y los atributos de la estructura del ecosistema forestal circundante.

Presencia de avifauna. Para caracterizar la población de C. magellanicus y de su ensamble avifaunístico, se empleó el método de Blondel et al. (1981), desarrollándose un total de 50 estaciones de escucha, separadas cada $150 \mathrm{~m}$ y con un radio de $40 \mathrm{~m}$, las cuales fueron ubicadas siguiendo los trazados de los senderos "Piedra de Águila" (4.430 m de longitud, con 29 estaciones de escucha), "Casa de Piedra" (1.280 m de longitud, con ocho estaciones de escucha), "El Aguilucho" ( $1.580 \mathrm{~m}$ de longitud, con 10 estaciones de escucha) y "Camino del Árbol" (530 m de longitud, con 3 estaciones de escucha) (figura 1). El horario para iniciar observaciones fue de 15 minutos después del amanecer, con el fin de hacer coincidir el muestreo con las horas de mayor actividad de las aves (Rozzi et al. 1995), extendiéndose hasta aproximadamente el medio día. Para capturar información al interior de cada estación de escucha, se realizaron tres repeticiones, en días no consecutivos, siempre siguiendo el mismo itinerario, en cada época del año estudiada: tres en repeticiones en primavera (octubre de 2008) y tres repeticiones en verano (marzo de 2009); es decir, seis repeticiones en total. Las estaciones de escucha tuvieron una duración de cinco minutos, pero se destinaron tres minutos antes de iniciar las evaluaciones, con el fin de es- 
tabilizar el comportamiento de la avifauna y las especies de avifauna detectadas durante este período fueron obviadas. En el caso de avistar o escuchar un individuo de la especie en estudio entre dos estaciones de escucha consecutivas, éstos se registraron en la estación de escucha anterior; sin embargo, cuando se produjo un cambio en la composición del bosque entre dos estaciones de escucha consecutivas, las especies se registraron en la estación que correspondió según las características de la vegetación donde se avistó o escuchó el o los individuos. Al interior de cada estación de escucha se registraron datos ambientales (coordenadas UTM, altitud, pendiente, exposición y condición atmosférica) y datos relacionados con C. magellanicus y su ensamble (especie, número de individuos observados), así como también datos de la composición y estructura de los bosques que ocupa C. magellanicus (tipo de bosque y especie arbórea que utiliza). Se caracterizó además la población de C. magellanicus y su ensamble mediante los siguientes parámetros poblacionales (Krebs 1985): riqueza de especies, abundancia relativa, índice de Shannon-Wiener, diversidad máxima e índice de equiparabilidad.

Atributos del ecosistema boscoso. Se evaluó la composición de los ecosistemas forestales identificando las especies dominantes en cada estación de escucha, aplicando criterios fisionómicos y cobertura de copa. Para determinar las características estructurales de los ecosistemas forestales se muestreó cada estación de escucha mediante el método propuesto por Prodan et al. (1997), el que define un tipo de unidad muestral circular no probabilística, evaluándose al interior de cada una de ellas diámetro a la altura del pecho (DAP), densidad de árboles (DAP $\geq 10 \mathrm{~cm})$, área

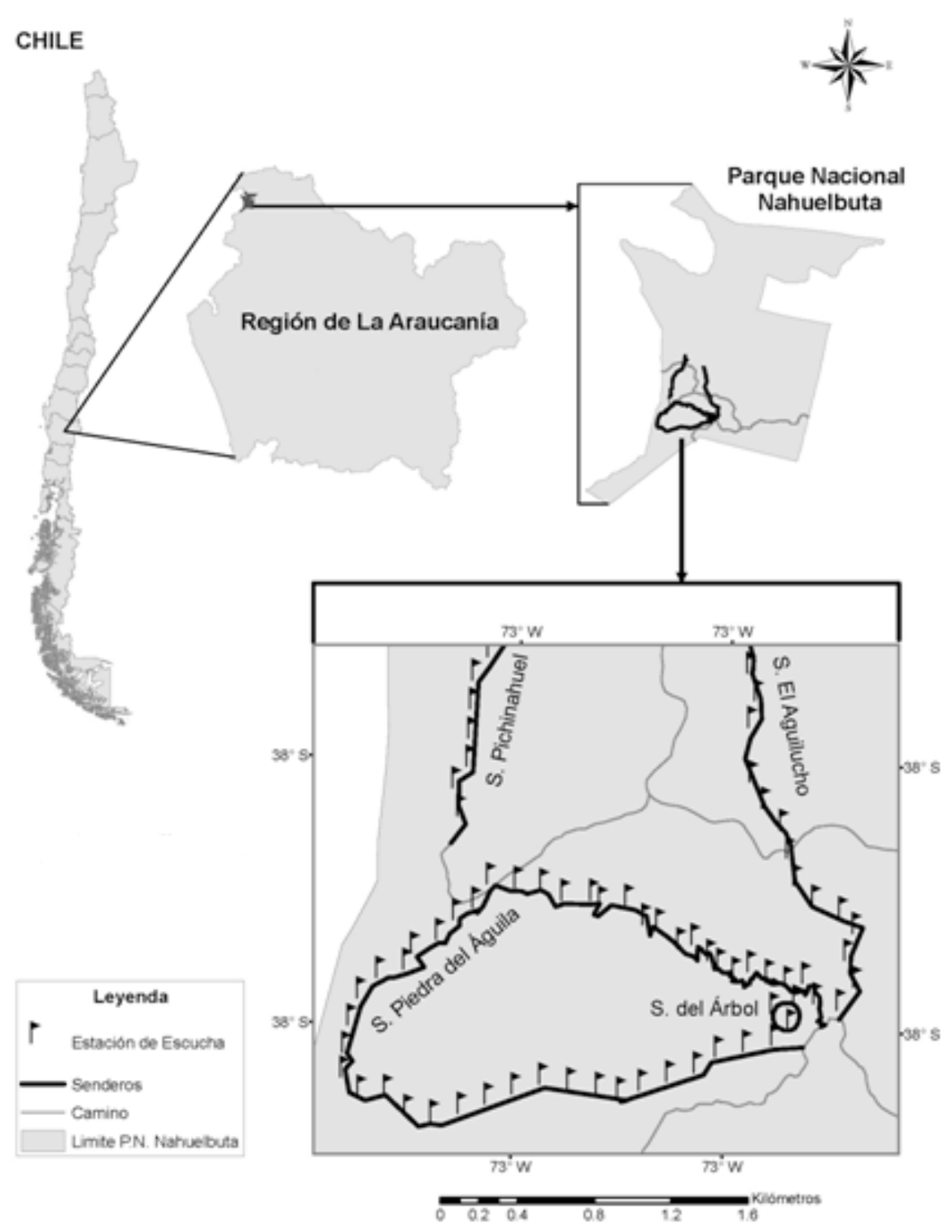

Figura 1. Localización geográfica del área de estudio dentro del parque nacional Nahuelbuta.

Geographical location of the study area within Nahuelbuta national park. 
basal y altura total. Para complementar esta información, se desarrolló un muestreo estructural-ecológico, mediante parcelas de área mínima de $120 \mathrm{~m}^{2}$ (Mueller-Dombois y Ellenberg 1974), al interior de las cuales se evaluó el tipo y número de estratos y la cobertura de copa de las especies arbóreas (DAP $\geq 10 \mathrm{~cm}$ ). A estas variables se agregaron otras, consideradas como de "exclusiva importancia" para la avifauna (Estades 2007), como composición florística, estado de descomposición de los árboles en pie (British Columbia Ministry of Forest 1995) y número de árboles.

Análisis estadístico. Se desarrolló un análisis exploratoriodescriptivo de los datos muestrales (Zar 1999), separándolos en una primera etapa en variables discretas y continuas, generando una descripción estadística de los datos, considerando media, desviación estándar, máximo y mínimo de cada variable. También se separaron entre variables medioambientales (condición atmosférica, pendiente, exposición, altitud y coordenadas geográficas), variables relacionadas con el bosque (tipo de bosque, especies dominantes, densidad, área basal, altura total, cobertura por estrato, DAP, diámetro medio cuadrático, estado de descomposición de los árboles en pie y número de estratos) y variables relacionadas con la avifauna (presencia de especies al interior de estaciones de escucha). En una segunda etapa, se desarrolló el análisis estadístico de los datos, utilizándose para el caso de las variables continuas la prueba t de Student, con el fin de probar la igualdad de medias, y la prueba de Levene, para la igualdad de varianzas. Para el caso de las variables discretas, se generaron tablas de contingencia, para luego aplicar pruebas de bondad de ajuste con la distribución de Chi-cuadrado de Pearson y el test exacto de Fisher (William 1993). Para poner a prueba la hipótesis planteada en el estudio, se estableció un nivel de significancia de $P<0,1$ y $P<0,05$, lo que indica un nivel de confiabilidad de un $95 \%$ y $99 \%$, respectivamente. Para el análisis de datos se usó el software SPSS 17.0.

\section{RESULTADOS}

Caracterización estructural de los bosques en que está presente C. magellanicus. Bosque de coigüe-araucaria. Los bosques de coigüe (Nothofagus dombeyi Mirb. Oerst.) y araucaria (Araucaria araucana Molina K. Koch), representaron un $48 \%$ del total de los puntos muestreados, siendo los más comunes en la zona de estudio, abarcando más del $25 \%$ de la superficie total del parque nacional Nahuelbuta. Estos bosques son los más oscuros y húmedos del parque nacional Nahuelbuta (cuadro 1), ya que la cobertura de copas del estrato dominante alcanza un $48 \%$ en promedio y están dominados por coigüe. En su estrato arbustivo destacaron especies como: coligüe (Chusquea culeou Desvaux.), sauco del diablo (Pseudopanax laetevirens Gay Frodin.), clavel del campo (Mutisia spinosa R. et Pav.), chinchín (Azara microphylla Hook. f.) y berberis (Berberis linearifolia Kunze ex Poepp. et Endl.).

Cuadro 1. Composición y estructura de la vegetación en los cuatro ecosistemas estudiados.

Composition and structure of vegetation in the four ecosystems studied.

\begin{tabular}{|c|c|c|c|c|c|}
\hline $\begin{array}{l}\text { Tipo de } \\
\text { ecosistema }\end{array}$ & $\begin{array}{l}\text { Especies representativas } \\
\text { por estrato }(*)\end{array}$ & $\begin{array}{l}\text { Cobertura de } \\
\text { copas estrato } \\
\text { dominante } \\
(\%)\end{array}$ & $\begin{array}{l}\text { Altura promedio } \\
\text { árboles } \\
\text { dominantes } \\
\text { (m) }\end{array}$ & $\begin{array}{l}\text { DAP promedio } \\
\text { árboles } \\
\text { dominantes } \\
\quad(\mathrm{cm})\end{array}$ & $\begin{array}{l}\text { Participación } \\
\text { por } \\
\text { especie } \\
(\%)\end{array}$ \\
\hline $\begin{array}{l}\text { Coigüe- } \\
\text { araucaria }\end{array}$ & $\begin{array}{l}\text { Em: coigüe; D y CD: araucaria, coigüe; } \\
\text { I: coigüe, araucaria; Ia: canelo, canelo } \\
\text { enano, quila, coligüe, tihuen, sauco del } \\
\text { diablo, chaura, taique, clavel del campo, } \\
\text { michay, chinchín, berberis. }\end{array}$ & 48 & $\begin{array}{c}30(\mathrm{Co}) \\
15-18(\mathrm{Ar})\end{array}$ & $\begin{array}{l}90(\mathrm{Co}) \\
150(\mathrm{Ar})\end{array}$ & $\begin{array}{l}70,4(\mathrm{Co}) \\
29,6(\mathrm{Ar})\end{array}$ \\
\hline $\begin{array}{l}\text { Coigüe } \\
\text {-araucaria- } \\
\text { lenga }\end{array}$ & $\begin{array}{l}\text { Em y D:coigüe, araucaria; } \\
\text { CD,I: coigüe, araucaria, lenga; Ia: cane- } \\
\text { lo enano, tihuen, chaura, chaura común, } \\
\text { taique, leñadura, saloll, racoma. }\end{array}$ & 55,5 & $\begin{array}{l}24(\mathrm{Co}) \\
22(\mathrm{Ar})\end{array}$ & $\begin{array}{c}105(\mathrm{Ar}) \\
75(\mathrm{Co}, \mathrm{Le})\end{array}$ & $\begin{array}{c}52,4(\mathrm{Le}) \\
28,6(\mathrm{Ar}) \\
19(\mathrm{Co})\end{array}$ \\
\hline Coigüe & $\begin{array}{l}\text { Em: coigüe; D: coigüe; Ia: canelo, che- } \\
\text { quén, quila argentina, taique, leñadura. }\end{array}$ & 32,8 & $22(\mathrm{Co})$ & $105(\mathrm{Co})$ & 100 (Co) \\
\hline Ñirre & $\begin{array}{l}\text { D: roble; I: roble, ñirre } \\
\text { Ia: ñirre, roble, araucaria. }\end{array}$ & 33 & 18 (Ro) & 90 (Co, Ar) & $\begin{array}{l}90,9(\tilde{\mathrm{Ni}}) \\
4(\mathrm{Co}) \\
2,8(\mathrm{Ar}) \\
2,3(\mathrm{Ro})\end{array}$ \\
\hline
\end{tabular}

Em: estrato emergente; D: estrato dominante; CD: estrato codominante; I: estrato intermedio; Ia: estrato inferior y arbustivo. (*) Nombres científicos en texto principal. 
Bosque de coigüe-arancaria-lenga. Este tipo de bosque está compuesto principalmente por coigüe, araucaria y lenga (Nothofagus pumilio Poepp. et Endl. Krasser), especies que en conjunto constituyeron el estrato dominante, cubriendo sus copas un 55,5\% de la superficie del bosque (cuadro 1). Como se puede apreciar en el cuadro 1, este tipo de bosque se divide en tres estratos bien demarcados: un estrato de árboles emergentes y dominantes cuyas especies principales son coigüe y araucaria, las que alcanzan hasta $24 \mathrm{~m}$ de altura (coigüe), en promedio, con diámetros que pueden superar los $100 \mathrm{~cm}$ (araucaria). Un segundo estrato, compuesto por especies arbóreas codominantes e intermedias, representadas por las mismas dos especies anteriores, a las cuales se les incorpora también la lenga; y un estrato inferior y arbustivo, donde es posible encontrar especies como canelo enano (Drimys andina Reiche R.A. Rodr. et Quez.), quila (Chusquea quila Kunth), tihuen (Chusquea sp. Kunth), chaura (Pernettya myrtilloides Sleumer D.J.Middleton), chaura común (Gaultheria phillyreifolia Pers Sleumer), taique (Desfontainea spinosa R. et Pav.), leñadura (Maytenus magellanica Lam. Hook. f.), saloll (Berberis serrato-dentata Lechl.) y racoma (Maytenus disticha Hook. f. Urban). En este estrato se encontró regeneración natural de algunas de las especies de árboles antes mencionadas, además del coirón (Festuca sp.), que es una especie representativa de los coironales ubicados en sectores más altos de los sitios muestreados en el presente estudio.

Bosque de coigüe. Este bosque resultó ser el más ralo de todos y posee tres estratos, con coigüe dominando los estratos emergente y dominante, alcanzando una altura promedio de $22 \mathrm{~m}$. No se pudo apreciar claramente en terreno la existencia de los estratos codominante ni intermedios. Los estratos inferior y arbustivo estuvieron compuestos por canelo (Drimys winteri J.R. et G. Forster) y chequén (Myrceugenia ovata Hook. et Arn. O. Berg), especies que se presentaron combinadas con quila argentina (Chusquea montana Kunth), taique y leñadura, entre otras especies. Exhibió un alto porcentaje de árboles muertos en pie y en el suelo del bosque, por lo que la oferta trófica para $C$. magellanicus resultó ser mayor que en los tipos de bosque anteriores (cuadro 1).

Bosque de ñirre. Este tipo de bosque se presentó estructuralmente caracterizado por tres estratos bien marcados (cuadro 1): un estrato dominante, donde el roble (Nothofagus obliqua Mirb. Oerst.) es la especie mejor representada, con alturas promedio que alcanzan los $18 \mathrm{~m}$, acompañada esporádicamente por individuos de gran diámetro (clase DAP $90 \mathrm{~cm}$ ) de coigüe y araucaria; un estrato intermedio en el cual las especies más comunes fueron ñirre (Nothofagus antarctica G. Forster Oerst.) y N. obliqua, las que en promedio pueden alcanzar entre 10 a $12 \mathrm{~m}$ de altura y con un $33 \%$ de cobertura de copas. El estrato inferior y arbustivo, correspondió fisionómicamente a un matorral achaparrado y estuvo conformado principalmente por
$N$. antarctica, la que alcanza un promedio de entre 4 a $6 \mathrm{~m}$ de altura total y una cobertura de copas de un $40 \%$, siendo la especie con la mayor participación porcentual en cuanto al número de individuos en este tipo de bosque. En este estrato, se pudieron observar individuos de regeneración natural de ñirre y, en menor escala, de roble y araucaria. En este mismo estrato, se apreciaron también individuos de especies arbustivas, como calafate (Berberis buxifolia Lam.), michay (Berberis darwinii Hook.), bácaris (Baccharis obovata Hook. et Arn.), chilco de Magallanes (Baccharis magellanica Lam. Pers.), además de algunas especies de gramíneas, como el coirón, las que en conjunto cubrieron cerca del $80 \%$ del piso del bosque. Todos los individuos, a excepción de $A$. araucana, presentaron algún grado de descomposición de los árboles en pie, ya sea solo en sus ramas o bien en parte o todo el fuste.

Caracterización de la población de Campephilus magellanicus y su ensamble avifaunistico. El ensamble avifaunístico del cual forma parte C. magellanicus estuvo representado por 30 especies (cuadros 2 y 3 ). Las mayores abundancias relativas promedio, considerando ambas temporadas analizadas (2008 y 2009, respectivamente), correspondieron a rayadito (Aphrastura spinicauda Gmelin, 1789), con $23,20 \%$ y 11,10 \%; cachaña (Enicognathus ferrugineus Muller, 1776), con 12,1 \% y 19,4\% y chucao (Scelorchilus rubecula Kittlitz, 1830), con 10,2 y 11,7 \%, en tanto que las menores abundancias relativas, excluyendo las especies rapaces, correspondieron a cachudito (Anairetes parulus Kittlitz, 1830), con $0 \%$ y 0,2 \%; tenca (Mimus thenca Molina, 1782), con $0,5 \%$ y $0 \%$ y zorzal (Turdus falklandii Quoy et Gaimard, 1824), con 0,5 \% y 0,5\%.

En relación a la riqueza específica, mediante los muestreo realizados en 2008 y 2009, se identificaron 19 y 26 especies, respectivamente. En cuanto a las especies menos representadas (cuadros 2 y 3 ), estas correspondieron especialmente al orden Falconiformes, entre las cuales se registraron: peuco (Parabuteo unicinctus Temminck, 1824), jote de cabeza negra (Coragyps atratus Bechstein, 1783), traro (Polyborus plancus Miller, 1777) y tiuque (Milvago chimango Vieillot, 1816). En el cuadro 4, se entregan cifras que ilustran la variación estacional de los parámetros poblacionales de C. magellanicus en los distintos ecosistemas forestales donde fue avistada la especie.

Del cuadro 4 se desprende que C. magellanicus está presente en todos los tipos de bosques, pero en primavera de 2008 fue más visible dentro del bosque de coigüe-araucaria; en cambio, en verano de 2009 se le vio más comúnmente dentro del bosque de ñirre.

En el año 2008, el bosque de coigüe-araucaria presentó la mayor riqueza de especies y la mayor abundancia relativa, en comparación con los otros tipos de bosque (cuadro 5). La segunda menor riqueza de especies se registró en el bosque de coigüe-araucaria-lenga, a pesar de que este tipo de bosque no es el que presentó los valores más bajos ni de riqueza ni abundancia relativa. 
Cuadro 2. Número de individuos (N) y abundancia relativa (AB\%) por especie. Parque Nacional Nahuelbuta (primavera 2008). Number of individuals $(\mathrm{N})$ and relative abundance (AB\%) by species. Nahuelbuta national park (spring 2008).

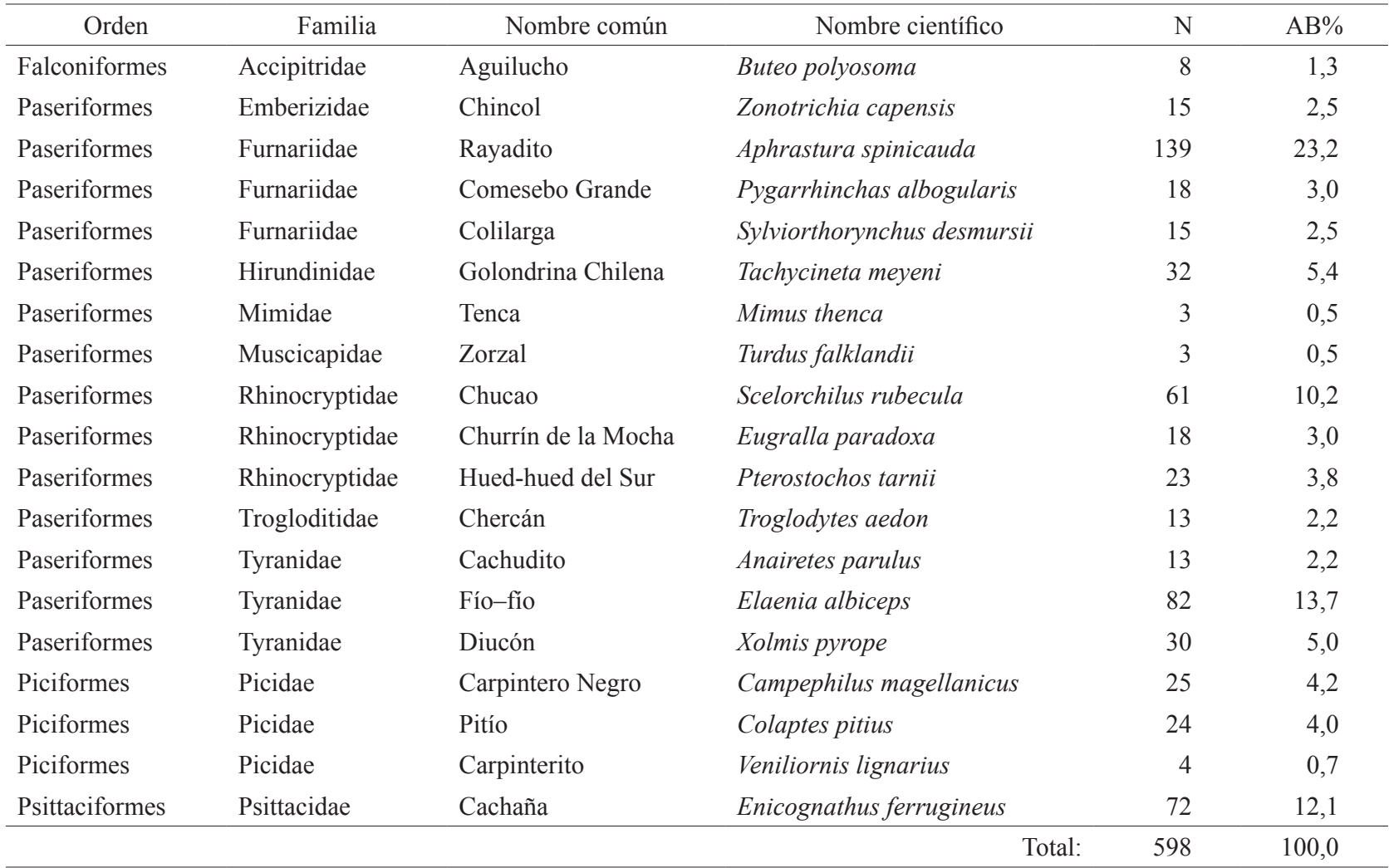

Cuadro 3. Número de individuos (N) y abundancia relativa (AB\%) por especie. Parque Nacional Nahuelbuta (verano de 2009). Number of individuals $(\mathrm{N})$ and relative abundance (AB\%) by species. Nahuelbuta national park (summer 2009).

\begin{tabular}{|c|c|c|c|c|c|}
\hline Orden & Familia & Nombre común & Nombre científico & $\mathrm{N}$ & $\mathrm{AB} \%$ \\
\hline Apodiformes & Trochilidae & Picaflor & Sephanoides galeritus & 179 & 21,2 \\
\hline Columbiformes & Columbidae & Torcaza & Patagioenas araucana & 17 & 2,0 \\
\hline Falconiformes & Accipitridae & Peuco & Parabuteo unicinctus & 1 & 0,1 \\
\hline Falconiformes & Cathartidae & Jote de Cabeza Negra & Coragyps atratus & 2 & 0,2 \\
\hline Falconiformes & Falconidae & Tiuque & Milvago chimango & 5 & 0,6 \\
\hline Falconiformes & Falconidae & Traro & Polyborus plancus & 4 & 0,5 \\
\hline Galliformes & Phasianidae & Codorniz $(*)$ & Callipepla californica & 3 & 0,4 \\
\hline Paseriformes & Emberizidae & Tordo & Curaeus curaeus & 4 & 0,5 \\
\hline Paseriformes & Emberizidae & Chincol & Zonotrichia capensis & 6 & 0,7 \\
\hline Paseriformes & Fringilidae & Jilguero & Sporagra barbata & 6 & 0,7 \\
\hline Paseriformes & Fringilidae & Cometocino Patagónico & Phrygilus patagonicus & 18 & 2,1 \\
\hline Paseriformes & Furnariidae & Rayadito & Aphrastura spinicauda & 94 & 11,1 \\
\hline Paseriformes & Furnariidae & Comesebo Grande & Pygarrhinchas albogularis & 74 & 8,7 \\
\hline Paseriformes & Hirundinidae & Golondrina Chilena & Tachycineta meyeni & 2 & 0,2 \\
\hline Paseriformes & Muscicapidae & Zorzal & Turdus falklandii & 4 & 0,5 \\
\hline Paseriformes & Rhinocryptidae & Churrín de la Mocha & Eugralla paradoxa & 12 & 1,4 \\
\hline Paseriformes & Rhinocryptidae & Hued- hued del sur & Pterostochos tarnii & 42 & 5,0 \\
\hline
\end{tabular}




\begin{tabular}{|c|c|c|c|c|c|}
\hline & & & & \multicolumn{2}{|c|}{ Conituación Cuadro 3} \\
\hline Paseriformes & Rhinocryptidae & Chucao & Scelorchilus rubecula & 99 & 11,7 \\
\hline Paseriformes & Rhinocryptidae & Churrín del Sur & Scytalopus magellanicus & 13 & 1,5 \\
\hline Paseriformes & Tyranidae & Cachudito & Anairetes parulus & 2 & 0,2 \\
\hline Paseriformes & Tyranidae & Viudita & Colorhamphus parvirostris & 19 & 2,2 \\
\hline Paseriformes & Tyranidae & Fío-fío & Elaenia albiceps & 35 & 4,1 \\
\hline Paseriformes & Tyranidae & Diucón & Xolmis pyrope & 18 & 2,1 \\
\hline Piciformes & Picidae & Carpintero Negro & Campephilus magellanicus & 17 & 2,0 \\
\hline Piciformes & Picidae & Pitío & Colaptes pitius & 7 & 0,9 \\
\hline Psittaciformes & Psittacidae & Cachaña & Enicognathus ferrugineus & 164 & 19,4 \\
\hline & & & Total: & 847 & 100,0 \\
\hline
\end{tabular}

$(*)$ Especie exótica, asilvestrada en Chile.

Cuadro 4. Variación estacional de los parámetros poblacionales de Campephilus magellanicus en distintos ecosistemas forestales del parque nacional Nahuelbuta.

Seasonal variation of population parameters of Campephilus magellanicus in different forest ecosystems at Nahuelbuta national park.

\begin{tabular}{lccccc}
\hline Parámetros poblacionales avifauna & Coigüe- araucaria & Coigüe-araucaria-lenga & Coigüe & Nirre & Total \\
\hline & \multicolumn{1}{c}{ Tipo de ecosistema (primavera de 2008) } & & & \\
\hline No de estaciones de escucha & 24 & 11 & 6 & 9 & 50 \\
Número de individuos & 9 & 3 & 2 & 3 & 17 \\
Frecuencia (\%) & 37,5 & 27,3 & 33,3 & 33,3 & 34 \\
\hline \multicolumn{1}{l}{ No de estaciones de escucha } & 24 & 11 & 6 & 9 & 50 \\
Número de individuos & 5 & 3 & 2 & 7 & 17 \\
Frecuencia (\%) & 10 & 6 & 4 & 14 & 34 \\
\hline
\end{tabular}

Cuadro 5. Parámetros poblacionales de la avifauna registrada en el estudio, desglosada por tipo de ecosistema y estación del año. Population parameters of birds recorded at the study area.

\begin{tabular}{|c|c|c|c|c|c|}
\hline \multirow{3}{*}{ Parámetros poblacionales } & \multicolumn{5}{|c|}{ Tipo de ecosistema } \\
\hline & Coigüe- araucaria & Coigüe-araucaria-lenga & Coigüe & Nirre & Total \\
\hline & \multicolumn{5}{|c|}{ Primavera de 2008} \\
\hline Abundancia $(\mathrm{N})$ & 284 & 120 & 70 & 121 & 594 \\
\hline Riqueza (S) & 17 & 15 & 14 & 16 & 19 \\
\hline Abundancia relativa ( $\mathrm{AB} \%)$ & 89 & 79 & 74 & 84 & 100 \\
\hline Diversidad (H') & 3,45 & 3,28 & 3,34 & 3,63 & 3,60 \\
\hline Diversidad máxima (H'máx) & 4,24 & 3,90 & 3,91 & 4,00 & 4,24 \\
\hline \multirow[t]{2}{*}{ Índice de equiparabilidad (E) } & 0,81 & 0,83 & 0,86 & 0,91 & 0,85 \\
\hline & \multicolumn{5}{|c|}{ Verano de 2009} \\
\hline Abundancia $(\mathrm{N})$ & 384 & 176 & 119 & 168 & 847 \\
\hline Riqueza de especies (S) & 20 & 15 & 14 & 22 & 26 \\
\hline Abundancia relativa ( $\mathrm{AB} \%)$ & 77 & 58 & 54 & 85 & 100 \\
\hline Diversidad (H') & 3,40 & 3,05 & 3,42 & 3,59 & 3,53 \\
\hline Diversidad máxima (H'máx.) & 4,32 & 3,90 & 3,81 & 4,46 & 4,70 \\
\hline Índice de equiparabilidad (E) & 0,78 & 0,78 & 0,90 & 0,81 & 0,75 \\
\hline
\end{tabular}


También se observó que en el año 2009 el bosque de ñirre presentó la mayor riqueza específica y que un $85 \%$ de los individuos registrados se encuentran en su interior, situación que se repitió en cuanto a diversidad y diversidad máxima (cuadro 5).

Relación entre la composición y estructura de los bosques con la composición con la presencia de C. magellanicus. Se apreció el uso evidente de esta especie por los bosques de coigüe-araucaria y ñirre $(F=23,8 \%$ y $23,7 \%$, respectivamente) (cuadro 6), debido a que estas especies satisficieron los requerimientos de hábitats de alimentación claves para C. magellanicus. La equiparabilidad fue similar en los cuatro tipos de bosque estudiados, lo que indica similares grados de parecido entre los valores de diversidad y diversidad máxima obtenidos en las cuatro comunidades avifaunísticas. En síntesis, se pudo establecer que las comunidades que presentaron una mayor ocurrencia de C. magellanicus son las mismas que exhibieron mayores valores en abundancia relativa y riqueza de especie y sus individuos estuvieron mejor repartidos entre las diferentes especies que la componen.

Descripción del hábitat de alimentación ocupado por C. magellanicus en el Parque Nacional Nahuelbuta. A partir del contenido del cuadro 7, se desprende que solo tres variables (cobertura estrato dominante, cobertura estrato herbáceo y estado de descomposición de los árboles en pie) se correlacionaron directamente con la presencia de C. magellanicus. Además, se observa que esta especie no seleccionó en forma diferencial las características estructurales de los bosques entre primavera y verano, excepto, el caso de la cobertura del estrato herbáceo, la que resultó ser muy significativa en primavera y significativa en verano. Mención especial merece la variable estado de descomposición de los árboles en pie, la que resultó estar muy significativamente correlacionada con la presencia de C. magellanicus.

\section{DISCUSIÓN}

Campephilus magellanicus se comporta como una especie ubicua al interior del parque nacional Nahuelbuta, siendo posible observarla en todos los tipos de ecosistemas estudiados. Allí conforma ensambles avifaunísticos compuestos por al menos unas 30 especies diferentes, cifra menor a las 44 especies que normalmente suelen encontrarse en los bosques templados (Rozzi et al. 1995). Considerando que existen notables diferencias en cuanto a composición y estructura (cuadro 1) entre los distintos ecosistemas estudiado, se puede inferir que C. magellanicus no exhibe el mismo grado de uso al interior de estos ecosistemas, porque cada uno de ellos posee singularidades que lo hacen más o menos adecuados para suplir sus requerimientos de hábitat. No obstante lo anterior, se puede aseverar que al interior del parque nacional Nahuelbuta, todos los ecosistemas estudiados poseen las condiciones de hábitat de alimentación necesarias para la existencia de $C$. magellanicus; en especial aquellos sectores donde dominan especies de Nothofagus.

Las diferencias en cuanto a la riqueza específica de especies de avifauna observada durante los muestreos del año 2008 (primavera) versus 2009 (verano), con 19 y 26 especies, respectivamente (cuadros 2 y 3 ), puede deberse a distintos factores, como las condiciones climáticas más favorables en 2009 (mayor temperatura y menores precipitaciones, en ambos casos, cifras promedio mensuales), lo que explica la aparición de especies como picaflor (Sephanoides galeritus Molina, 1782), torcaza (Patagioenas araucana Lesson, 1827) y codorniz (Callipepla californica Shaw, 1798), entre otras. Es importante señalar además que durante el muestreo de 2008 se encontraba la Dirección de Vialidad realizando obras de mejoras de caminos al interior del parque nacional Nahuelbuta, circunstancia que operó negativamente y que explica un menor número de especies de aves observadas.

Algunos parámetros demográficos secundarios exhiben variaciones dependiendo de la estación del año; en

Cuadro 6. Parámetros poblacionales y comunitarios promedio del ensamble de avifauna por tipo de ecosistema y estación del año.

Population and community parameters of birds recorded at the study area.

\begin{tabular}{|c|c|c|c|c|c|c|c|}
\hline \multirow{2}{*}{ Tipos de ecosistema } & \multicolumn{7}{|c|}{$\begin{array}{l}\text { Parámetros poblacionales y comunitarios promedio } \\
\text { del ensamble de avifauna (primavera/verano) }(*)\end{array}$} \\
\hline & $\mathrm{F}$ & $\mathrm{N}$ & $\mathrm{S}$ & $\mathrm{AB}$ & $\mathrm{H}^{\prime}$ & H’ máx. & $\mathrm{E}$ \\
\hline Coigüe-araucaria & 23,8 & 334 & 18 & 83 & 3,42 & 4,28 & 0,79 \\
\hline Coigüe-araucaria- lenga & 16,7 & 148 & 15 & 68 & 3,17 & 3,90 & 0,80 \\
\hline Coigüe & 18,7 & 94 & 14 & 64 & 3,38 & 3,86 & 0,88 \\
\hline Nirre & 23,7 & 144 & 19 & 84 & 3,61 & 4,23 & 0,86 \\
\hline
\end{tabular}

$\mathrm{F}=$ frecuencia (\%) (solo Campephilus magellanicus); $\mathrm{N}=$ abundancia; $\mathrm{S}=$ riqueza; $\mathrm{AB}=$ abundancia relativa (\%); $\mathrm{H}^{\prime}=$ diversidad de ShannonWiener; H’máx. = diversidad máxima; $\mathrm{E}=$ índice de equiparabilidad; $(*)$ : cifras redondeadas. 
Cuadro 7. Variables relacionadas con la presencia de Campephilus magellanicus en el Parque Nacional Nahuelbuta.

Variables related to the presence of Campephilus magellanicus at Nahuelbuta national park.

\begin{tabular}{|c|c|c|}
\hline Variables continuas & $\begin{array}{c}\text { Valor } P\left({ }^{a}\right) \\
\text { (primavera de } 2008)\end{array}$ & $\begin{array}{c}\text { Valor } P\left({ }^{b}\right) \\
\text { (verano de } 2009 \text { ) }\end{array}$ \\
\hline Altitud (m s.n.m.) & $0,475 \mathrm{~ns}$ & $0,524 \mathrm{~ns}$ \\
\hline Pendiente $(\%)$ & $0,721 \mathrm{~ns}$ & $0,754 \mathrm{~ns}$ \\
\hline Densidad (árboles ha-1) & $0,146 \mathrm{~ns}$ & $0,193 \mathrm{~ns}$ \\
\hline Área basal $(\mathrm{AB})\left(\mathrm{m}^{2} \mathrm{ha}^{-1}\right)$ & $0,112 \mathrm{~ns}$ & $0,157 \mathrm{~ns}$ \\
\hline Altura total promedio $(\mathrm{HT})(\mathrm{m})$ & $0,478 \mathrm{~ns}$ & $0,475 \mathrm{~ns}$ \\
\hline Cobertura estrato emergente $(\%)$ & $0,321 \mathrm{~ns}$ & $0,278 \mathrm{~ns}$ \\
\hline Cobertura estrato dominante $(\%)$ & $0,076^{*}$ & $0,063^{*}$ \\
\hline Cobertura estrato inferior y arbustivo (\%) & $0,907 \mathrm{~ns}$ & $0,906 \mathrm{~ns}$ \\
\hline Cobertura estrato herbáceo $(\%)$ & $0,042 * *$ & $0,066^{*}$ \\
\hline Forrajeo (\%) & $0,235 \mathrm{~ns}$ & $0,238 \mathrm{~ns}$ \\
\hline Diámetro medio cuadrático $(\mathrm{DMC})(\mathrm{cm})$ & $0,451 \mathrm{~ns}$ & $0,430 \mathrm{~ns}$ \\
\hline Variables discretas & Valor $P\left({ }^{c}\right)$ & \\
\hline Tipo de bosque & $0,833 \mathrm{~ns}$ & \\
\hline Exposición & $0,684 \mathrm{~ns}$ & \\
\hline Número de estratos & $0,373 \mathrm{~ns}$ & \\
\hline Presencia de coigüe & $0,963 \mathrm{~ns}$ & \\
\hline Presencia de araucaria & $0,180 \mathrm{~ns}$ & \\
\hline Presencia de lenga & $0,235 \mathrm{~ns}$ & \\
\hline Presencia de ñirre & $0,450 \mathrm{~ns}$ & \\
\hline Estado de descomposición de los árboles en pie & $0,026 * *$ & \\
\hline
\end{tabular}

*: Existe relación significativa entre variable indicada y presencia de $C$. magellanicus $(P<0,1)$.

**: Existe relación muy significativa entre variable indicada y presencia de $C$. magellanicus $(P<0,05)$.

ns: No existen diferencias significativas entre variable indicada y presencia de $C$. magellanicus.

${ }^{(a)}$ y $\left({ }^{b}\right)$ : prueba $t ;\left(^{c}\right)$ : prueba de $\chi^{2}$

efecto, en primavera de 2008, la especie más abundante en cualquier tipo de bosque correspondió al rayadito, en tanto que durante el verano de 2009 fue el picaflor (cuadros 2 y 3). Esta última circunstancia se debe muy posiblemente a la notable y profusa floración a la fecha del muestreo del quintral amarillo (Desmaria mutabilis Poepp. et Endl. Tieghem ex B.D. Jackson) (IFANOS 2000). Además, ciertas especies se presentan con mayor número de individuos y abundancia relativa, indistintamente se trate de primavera o verano, como es el caso de fío-fío (Elaenia albiceps d'Orbigny et Lafresnaye, 1837), cachaña y chucao.

Especies de avifauna como tenca y tordo (Curaeus curaeus Molina, 1782) presentan en el área de estudio las menores abundancias relativas, lo que se explica porque dichas especies están asociadas a otras estructuras, como por ejemplo, a bosques más abiertos (como los bosques de ñirre), o bien a formaciones vegetacionales más abiertas, como praderas o matorrales (Araya y Millie 1998).

Si se comparan los parámetros poblacionales y comunitarios de la avifauna por tipo de ecosistema y estación del año (cuadros 4 y 5), se puede apreciar que en el año 2008 (primavera), al interior de los bosques de coigüearaucaria se registró la mayor cantidad de individuos de C. magellanicus. Por ende, se encuentra aquí también la mayor frecuencia porcentual, lo cual confirma lo expuesto por Rodríguez (2001) en cuanto al uso de C. magellanicus de este tipo de bosques. En efecto, C. magellanicus encuentra aquí árboles que poseen características necesarias para su ocurrencia, debido a la dominancia de coigüe, que es la especie más usada por esta especie para hacer cavidades para usarlas como dormidero y/o nido. En contraste, el año 2009 (verano) se registró una mayor cantidad de 
individuos de C. magellanicus en bosques de ñirre, lo cual se debe a que la mayoría de los individuos de C. magellanicus detectados durante esa temporada se encontraban alimentándose en árboles de esta especie arbórea, que es hospedera de larvas de gusano de tebo (Chilecomadia valdiviana Philippi), las que son fáciles de extraer, debido al pequeño diámetro que presenta ñirre. Ello se debe al hábito de crecimiento del ñirre, especie que crece comúnmente como arbolito pequeño o arbusto achaparrado (Hoffmann 2005). Cabe hacer notar además que durante el verano es más numerosa la dotación de larvas de gusano de tebo en el parque nacional Nahuelbuta (Cerda et al. 2000).

Por su parte, en el año 2008, el bosque de coigüearaucaria es el que presenta la mayor riqueza de especies, abundancia y abundancia relativa de la avifauna, pero no así la mayor diversidad, sino que esta se encuentra en el bosque de ñirre (cuadro 5), lo cual podría deberse a que en este último tipo de bosque los individuos observados se reparten proporcionalmente entre las distintas especies que lo conforman (Begon et al. 2006), lo cual se ve refrendado por la mayor diversidad máxima que exhibe este tipo de ecosistema.

Además, al tratarse de bosques con una gran cobertura a nivel del sotobosque, se presentan condiciones de hábitat más adecuadas para las diferentes especies que habitan al interior de estas formaciones boscosas. El año 2009, en el bosque de ñirre se encontró también la mayor riqueza de especies y abundancia relativa de avifauna, lo que se debe a que son bosques más abiertos, permitiendo la llegada de aves típicas de matorrales y zonas abiertas, donde se encuentran sus áreas de alimentación como por ejemplo peuco, tiuque y codorniz (Araya y Millie 1998), especies que no se registraron al interior de otras formaciones boscosas. Los bosques de coigüe-araucaria poseen una menor diversidad, pero no presenta los valores más bajos de riqueza ni de abundancia relativa, por lo que indicaría que existe un reparto desigual de los individuos entre los distintos tipos de bosque.

Desde el punto de vista estructural, el bosque de coigüe-araucaria se destacó por poseer grandes árboles, los que sobrepasan fácilmente los $30 \mathrm{~m}$ de altura $\mathrm{y}$, en algunos casos, más de $150 \mathrm{~cm}$ de DAP. Además, presenta un alta proporción de árboles muertos en pie, lo que representa una buena oferta trófica para la avifauna presente en el parque nacional Nahuelbuta, especialmente para C. magellanicus, la que se alimenta preferentemente de larvas del coleóptero del coigüe (Cheloderus childreni Gray, 1832) (Cerda et al. 2000).

Debido a la gran mortalidad de individuos observada al interior del bosque de ñirre se genera una mayor oferta trófica para C. magellanicus, porque aparecen con mayor facilidad larvas e insectos, los cuales representan una fuente de alimento para esta especie durante los meses de primavera y verano.

En cuanto al hábitat de alimentación de C. magellanicus, el presente estudio establece que las variables es- tructurales más correlacionadas con presencia de C. magellanicus en el parque nacional Nahuelbuta corresponden a la cobertura de los estratos dominante y herbáceo, así como también al estado de descomposición de los árboles en pie. Ello no coincide con lo establecido por Rodríguez (2001), quien señala que el tipo de bosque es una variable clave para caracterizar el hábitat de alimentación de C. magellanicus. Si bien es cierto, dicha autora desarrolló sus estudios a escala de árboles individuales, sus resultados contrastantes sugieren el hecho que C. magellanicus, como cualquiera otra especie de avifauna, tiene sus requerimientos específicos para cada escenario ecológico, por lo que estos deben ser determinados en detalle en cada caso. El presente estudio establece también que la presencia de C. magellanicus en los ensambles avifaunísticos varía en función de la composición y estructura específica de los bosques estudiados, lo que coincide con lo planteado por Díaz et al. (2005).

En relación a las variables correlacionadas con la presencia de C. magellanicus en el parque nacional Nahuelbuta (cuadro 7), se puede establecer que C. magellanicus usa indistintamente en las dos estaciones estudiadas ecosistemas forestales que posean árboles en pie de un estado de descomposición más avanzado, hecho que concuerda con lo planteado por Schlatter y Vergara (2005). En efecto, dichos autores identifican esta misma variable como una de las más determinantes para la presencia de C. magellanicus, ya que los árboles en pie con un mayor grado de descomposición sirven como árboles-nido y corresponden también a árboles que poseen una mayor oferta trófica, encontrándose en estos individuos una mayor proporción de insectos y larvas de los cuales se pueden alimentar (Schlatter y Vergara 2005). Si se hubieran hecho comparaciones más contrastadas, como invierno versus verano, lo más probable es que se hubiera detectado una estacionalidad marcada en el ensamble avifaunístico del cual forma parte C. magellanicus (Ibarra et al. 2010).

A base de los resultados obtenidos en el presente estudio, se puede establecer que la cobertura de la vegetación es una característica clave del hábitat de alimentación de C. magellanicus; en el caso de la cobertura del estrato herbáceo, su importancia se debe a una mayor oferta trófica disponible para esta especie, considerando que C. magellanicus se alimenta de frutos de especies vegetales como Berberis serrato-dentata y Maytenus spp, así como también de hormigas, ortópteros, blattáridos, pseudoescorpiones y oribátidos (Ojeda 2003), todos los cuales están disponibles en este estrato. La cobertura del estrato dominante es otra característica clave del hábitat que ocupa C. magellanicus, lo cual se debe a que en bosques de una gran cobertura presentan mejores condiciones de refugio para los polluelos, así como también protección ante cambios acentuados en las condiciones del clima (Ojeda 2004). Además, la correlación con el estrato dominante señala una necesidad de protección contra posibles depredadores, especialmente debido al hecho que C. magellani- 
cus baja en ocasiones hasta el piso del bosque en busca de alimentación.

En el presente estudio exploratorio, se establece claramente que al interior del parque nacional Nahuelbuta, C. magellanicus utiliza preferentemente el coigüe para anidar, resultado que concuerda con lo observado por Rodríguez (2001), y el ñirre para alimentarse (cuadro 6). También se comprueba que la presencia de C. magellanicus está estrechamente relacionada con una alta biodiversidad de aves, lo que la refrenda como especie paraguas y que las comunidades avifaunísticas con mayor ocurrencia de C. magellanicus poseen también los mayores valores en parámetros poblacionales, por lo que es una especie bioindicadora de bosques en buen estado de conservación de los ecosistemas forestales que habita.

\section{CONCLUSIONES}

Los resultados obtenidos concuerdan con lo planteado en la hipótesis de trabajo, porque se comprobó que en los ecosistemas forestales del parque nacional Nahuelbuta existen atributos estructurales de la vegetación que están correlacionados directamente con el hábitat de alimentación de C. magellanicus, como es el caso de la cobertura del estrato herbáceo y el estado de descomposición de los árboles en pie. De igual forma, se comprobó que no existen variaciones estacionales de estos atributos de la estructura de la vegetación, puesto que, indistintamente del año y estación de que se trate (2008, condición de primavera versus 2009, condición de verano), siempre resultaron estar correlacionados los mismos atributos con el hábitat de alimentación de C. magellanicus.

Se comprueba la existencia de una correlación positiva entre la cobertura del estrato dominante de la vegetación y la presencia de C. magellanicus, lo que sugiere que la existencia de bosques con una gran cobertura presentan mejores condiciones para C. magellanicus, puesto que al interior de estos es mayor el grado de protección para los polluelos ante cambios acentuados en las condiciones del clima, así como también para los individuos adultos, contra el ataque de posibles depredadores, como aguilucho chico (Buteo albigula Philippi, 1899), peuquito (Accipiter bicolor Philippi y Landbeck, 1864), traro (Caracara plancus Miller, 1777) y rapaces nocturnas, probablemente del orden Strigiformes.

\section{AGRADECIMIENTOS}

Los autores agradecen a los proyectos DIUFRO DI110048 y FONDEF D10I1038, por aportar los recursos financieros que hicieron posible la presente publicación. También, se agradece a los Sres. Ricardo González, académico del Departamento de Ciencias Forestales de la Universidad de La Frontera y Marcelo Saavedra, profesional de la Corporación Nacional Forestal de la región de La Araucanía, por sus aportes al presente trabajo.

\section{REFERENCIAS}

Aguayo M, A Pauchard, G Azócar, O Parra. 2009. Cambio del uso del suelo en el centro sur de Chile a fines del siglo XX. Entendiendo la dinámica espacial y temporal del paisaje. Revista Chilena de Historia Natural 82(3): 361-374.

Angelstam P, G Mikusinski. 1994. Woodpeckers assemblages in natural and managed boreal and hemiboreal forest - a review -. Annales Zoologici Fennici31(1):157-172.

Arango X, R Rozzi, F Massardo, F Anderson, C Ibarra. 2007. Descubrimiento e implementación del Pájaro Carpintero Gigante (Campephilus magellanicus) como especie carismática: una aproximación biocultural para la conservación en la Reserva de Biósfera Cabo de Hornos. Magallania 35(2):71-88.

Araya B, G Millie. 1998. Guía de Campo de las Aves de Chile. Santiago, Chile. Editorial Universitaria. 406 p.

British Columbia Ministry of Forest. 1995. Biodiversity Guidebook. Forest Practices Code of British Columbia. Vancouver, Canada. British Columbia Ministry of Forest. 99 p.

Begon M, C Towsend, J Harper.2006. Ecology. From Individuals to Ecosystems. 4th Edition. London, U.K. Blackwell Publishing. $759 \mathrm{p}$.

Blondel J, C Ferry, B Frochat. 1981. Points counts with unlimited distance. Studies in Avian Biology 6(1): 414-420.

Cerda L, A Angulo, A Durán, T Olivares. 2000. Insectos asociados a bosques del centro sur de Chile. In: Baldini A, L Pancel eds. Agentes de daño en el bosque nativo. Santiago, Chile. Editorial Universitaria. p. $201-280$.

Chazarreta M, L Ojeda, A Trejo. 2011. Division of labour in parental care in the Magellanic Woodpecker Campephilus magellanicus. Journal of Ornithology (152)2: 231-242.

Czeszczewik D, W Walankiewicz. 2006. Logging affects the white-backed woodpecker Dendrocopus leucotos distribution in Bialowiesa Forests. Annales Zoologici Fennici 43(2): 221-227.

Díaz I, J Armesto, S Reid, K Sieving, M Willson. 2005. Linking forest structure and composition: avian diversity in sucessional forests of Chiloé Island, Chile. Biological Conservation 123(1): 91-101.

Drever M, K Aitken, A Norris, K Martin. 2008. Woodpeckers as reliable indicators of bird richness, forest health and harvest. Biological Conservation 141(3): 624-634.

Estades C. 2007. Manejo de Fauna Silvestre. In Hernández J, C de la Maza, C Estades eds. Biodiversidad: Manejo y Conservación de Recursos Forestales. Santiago, Chile. Editorial Universitaria. p. 544-566.

Hoffman A. 2005. Flora silvestre de Chile. Zona Araucana. Quinta Edición. Santiago, Chile. Ediciones Fundación Claudio Gay. 257 p.

Ibarra JT, T Altamirano, N Gálvez, I Rojas, J Laker, C Bonacic. 2010. Avifauna de los bosques templados de Araucaria araucana del sur de Chile. Ecología Austral 20(1):33-45.

IFANOS (Institute for Applied Ecological Studies, DE). 2000. Catastro de Flora y Fauna en el Parque Nacional Nahuelbuta para la Corporación Nacional Forestal (CONAF) IX Región Informe Final. Temuco/Osorno. Chile. 47 p.

IUCN (Unión Internacional para la Conservación de la Naturaleza, ES). 2014. The IUCN Red List of Threatened Species. Version 2014.2. The International Union for Conservation of Nature (IUCN). Consultado 05 ago. 2014. Disponible en 
http://www.iucnredlist.org.

Krebs Ch. 1985. Ecología. Estudio de la distribución y la abundancia. México D. F., México. Editorial Harla. 753 p.

Mikusinski G, M Gromadzki, P Chylarecki. 2001. Woodpeckers as indicators of forest bird diversity. Conservation Biology 15(1): 208-217.

Mueller-Dombois D, Ellenberger H. 1974. Aims and methods of vegetation ecology. New York, United States. John Wiley and Sons. 547 p.

Ojeda V. 2003.Magellanic Woodpecker frugivory and predation on a lizard. The Wilson Bulletin 115(2): 208-210.

Ojeda V. 2004.Breeding biology and social behaviour of Magellanic Woodpeckers (Campephilus magellanicus) in Argentine Patagonia. European Journal of Wildlife Research 50(1): 18-24.

Ojeda V, L Chazarreta. 2006. Provisioning of Magellanic Woodpecker (Campephilus magellanicus) nestlings with vertebrate prey. The Wilson Journal of Ornithology 118(2):251-254.

Ojeda V, M Suárez, T Kitzberger. 2007. Crown dieback events as key processes creating cavity habitat for magellanic woodpeckers. Austral Ecology 32(4): 436-445.

Prodan M, R Peters, F Cox, P Real. 1997. Mensura Forestal. San José, Costa Rica. Imprenta Instituto Interamericano de Cooperación para la Agricultura (IICA). 572 p.

Rodríguez S. 2001. Análisis preliminar de cavidades arbóreas de Carpintero Negro (Campephilus magellanicus) en el Parque Nacional Nahuelbuta, IX Región, Chile. Tesis Médico Veterinario. Chillán, Chile. Facultad de Medicina Veterinaria, Universidad de Concepción. 59 p.
Rozzi R, D Martínez, M Willson, C Sabag. 1995. Avifauna de los bosques templados de Sudamérica. In Armesto J, C Villagrán, MK Arroyo eds. Ecología de los Bosques Nativos. Santiago, Chile. Editorial Universitaria. p. 135-154.

Schlatter R, P Vergara. 2005. Magellanic Woodpecker (Campephilus magellanicus) sap feeding. J Ornithology 146(2): 188-190.

Short L. 1970.Habits and relationships of the Magellanic Woodpecker.The Wilson Bulletin 82(2):115- 129.

Vergara P, R Schlatter. 2004. Magellanic Woodpecker (Campephilus magellanicus) abundance. Journal of Ornithology 145(4): 343-351.

Vergara P, R Schlatter. 2006. Aggregate retention in two Tierra del Fuego Nothofagus Forests: Short term effects on bird abundance. Forest Ecology and Management 225(1-3): 213-224.

Virkkala, R. 2006. Why study woodpeckers? The significance of woodpeckers in forest ecosystems. Annales Zoologici Fennici 43(3): 82-85.

Walpole M, N Leader-Williams. 2002. Tourism and flagship species in conservation. Biodiversity and Conservation 11(3): 543-547.

Williams B. 1993. Biostatistics. London, UK. Chapman and Hall Ltd. $201 \mathrm{p}$

Willson M, T De Santo, C Sabag, J Armesto. 1994. Avian communities of fragmented South-temperate rainforest in Chile. Conservation Biology 8(2): 508-520.

Zar J. 1999. Biostatistical Analysis. New York, U.S.A. PrenticeHall. 718 p. 